Supporting Information:

\title{
Stacking Engineering: A Boosting Strategy for 2D Photocatalysts
}

Jinfeng Zhao, ${ }^{1}$ Yanliang Zhao, ${ }^{1}$ Huijie He, ${ }^{1}$ Panwang Zhou,,${ }^{1,}$ Yan Liang, $, 2,{ }^{*}$ Thomas Frauenheim $, 3,4{ }^{*}$

${ }^{1}$ Institute of Molecular Sciences and Engineering, Institute of Frontier and Interdisciplinary Science, Shandong University, Qingdao 266237, China;

${ }^{2}$ Bremen Center for Computational Materials Science, University of Bremen, 28359 Bremen, Germany;

${ }^{3}$ Beijing Computational Science Research Center, 100193 Beijing, P.R. China;

${ }^{4}$ Shenzhen JL Computational Science and Applied Research Institute, 518109 Shenzhen, P.R. China.

*Corresponding author: pwzhou@,sdu.edu.cn (P.Z.); yliang@uni-bremen.de (Y.L.);

thomas.frauenheim@bccms.uni-bremen.de (T.F.) 

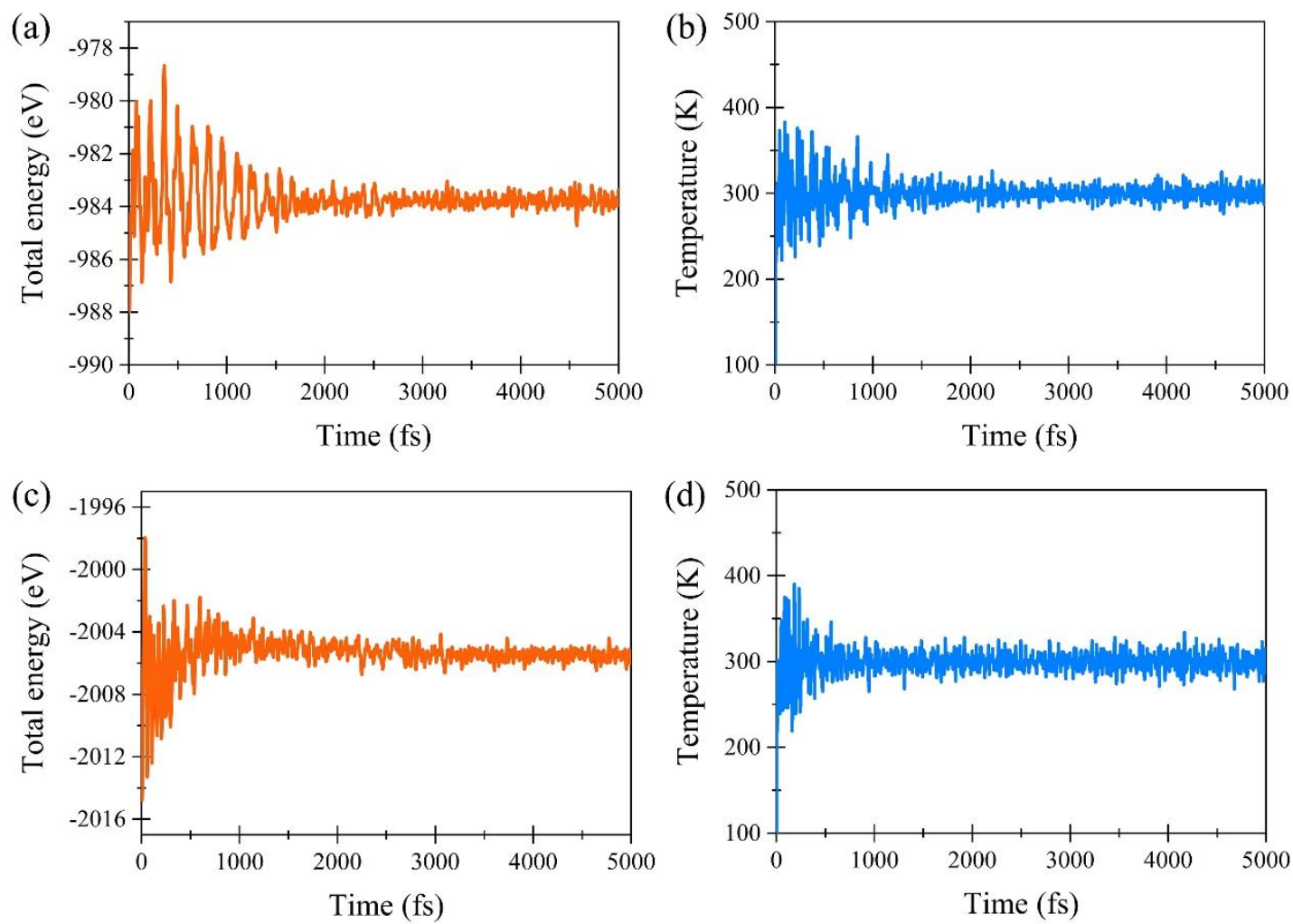

Figure S1. The fluctuation of total energy and temperature as a function of time obtained from adiabatic molecular dynamical simulations for (a), (b) monolayer $\mathrm{MoSi}_{2} \mathrm{~N}_{4}$ and (c), (d) $\mathrm{AB}-\mathrm{MoSi}_{2} \mathrm{~N}_{4}$ at $300 \mathrm{~K}$.
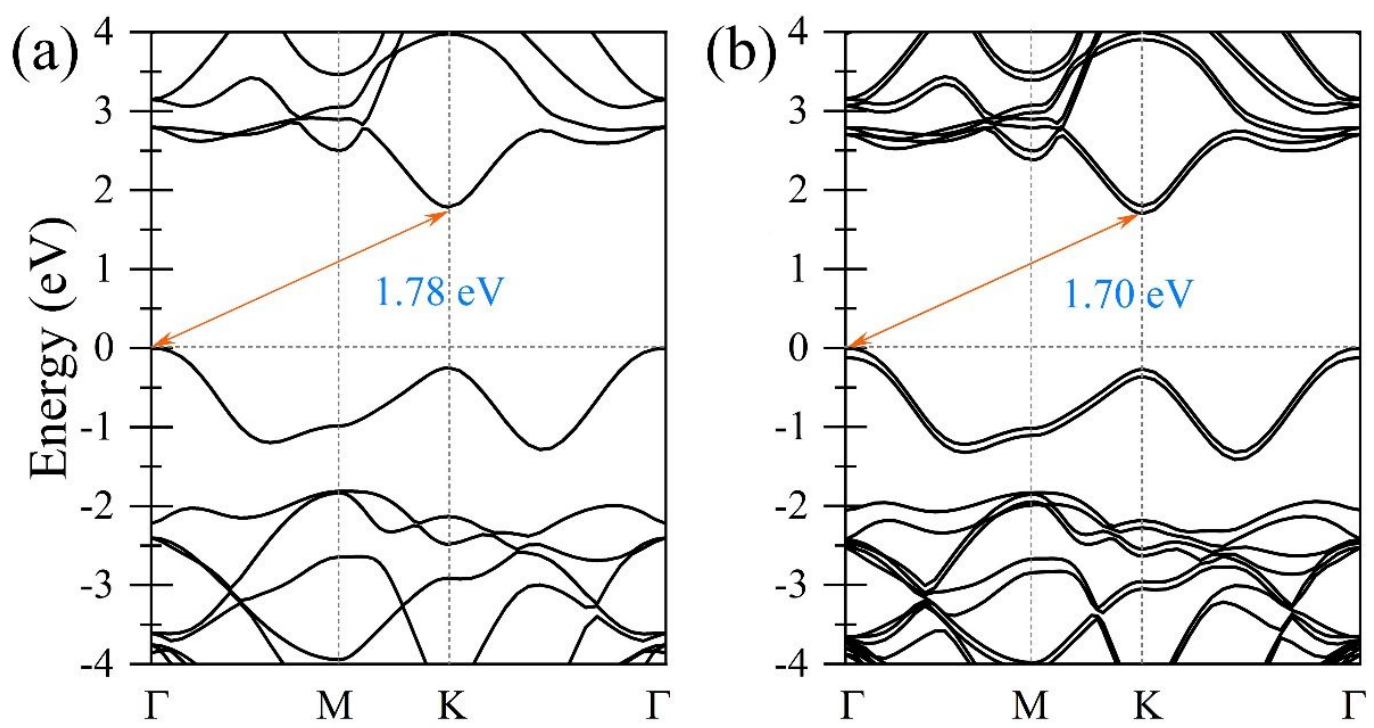

Figure S2. Band structures of (a) monolayer and $\mathrm{AB}-\mathrm{MoSi}_{2} \mathrm{~N}_{4}$ at PBE functional. 


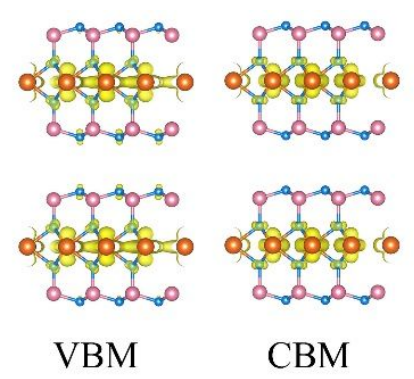

AA
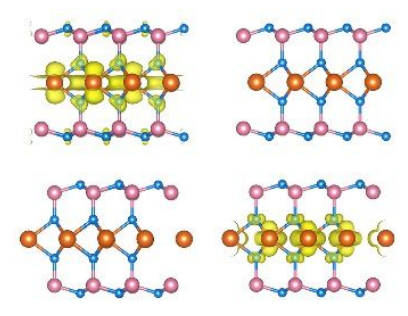

VBM CBM

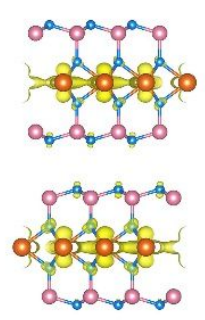

VBM
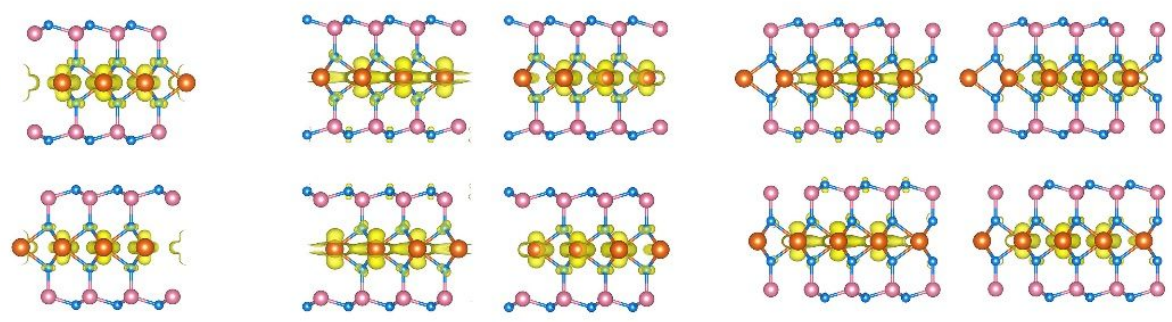

CBM

$\mathrm{AA}^{*}$

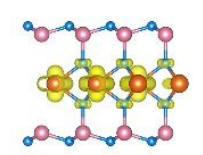

CBM

$\mathrm{AB}$

Figure S3. Distributions of CBM and VBM for five different highly symmetric bilayer $\mathrm{MoSi}_{2} \mathrm{~N}_{4}$ with $\mathrm{AA}, \mathrm{AA}^{*}, \mathrm{AB}, \mathrm{AB}^{*}, \mathrm{AC}$ and $\mathrm{AC}^{*}$ stacking modes.

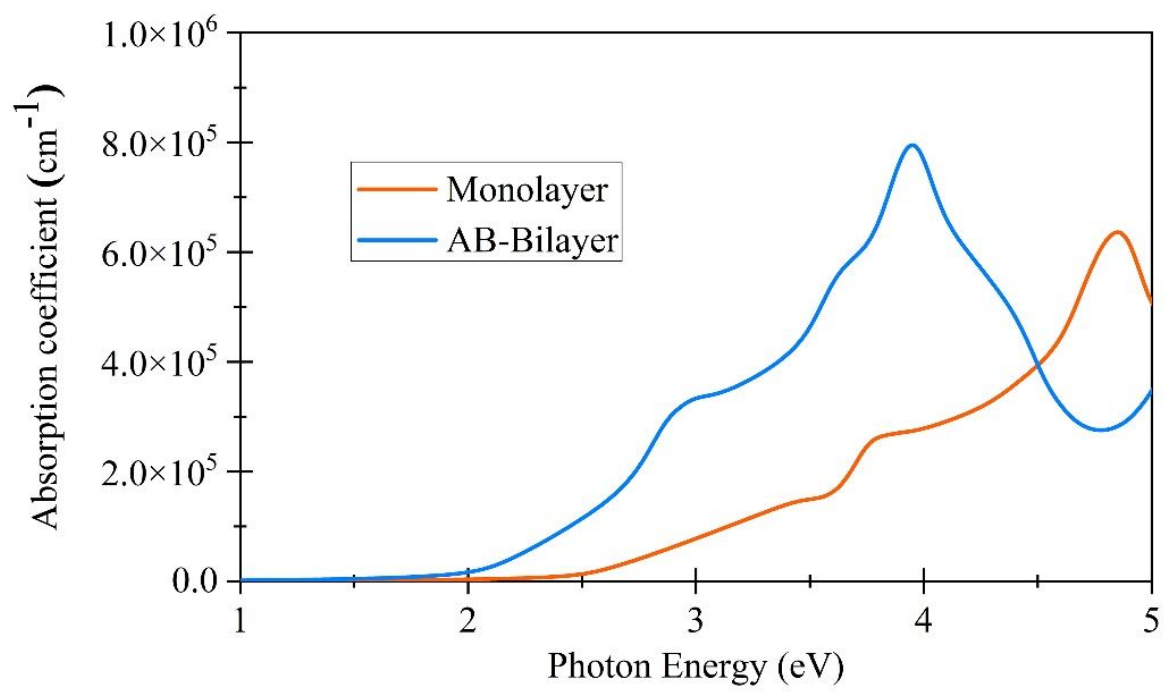

Figure S4. Optical absorption coefficients of monolayer and $\mathrm{AB}-\mathrm{MoSi}_{2} \mathrm{~N}_{4}$. 


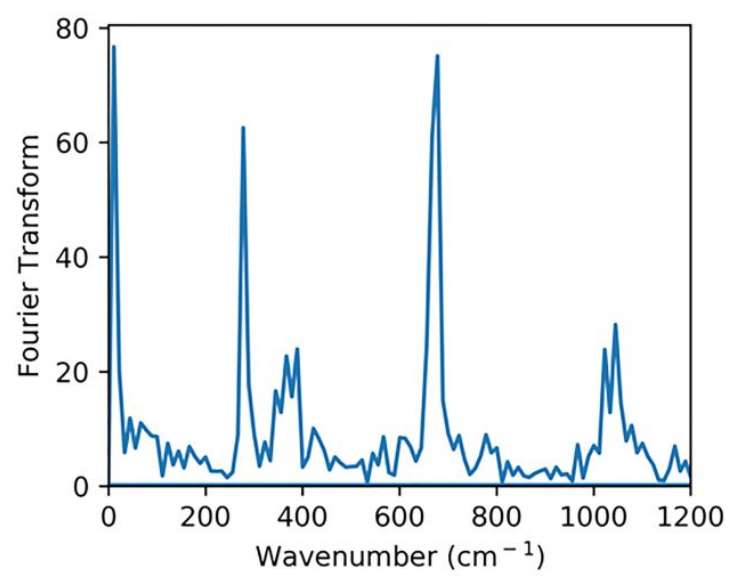

Figure S5. Fourier transform of the time-dependent state energy evolutions of VBM for $\mathrm{AB}-\mathrm{MoSi}_{2} \mathrm{~N}_{4}$.
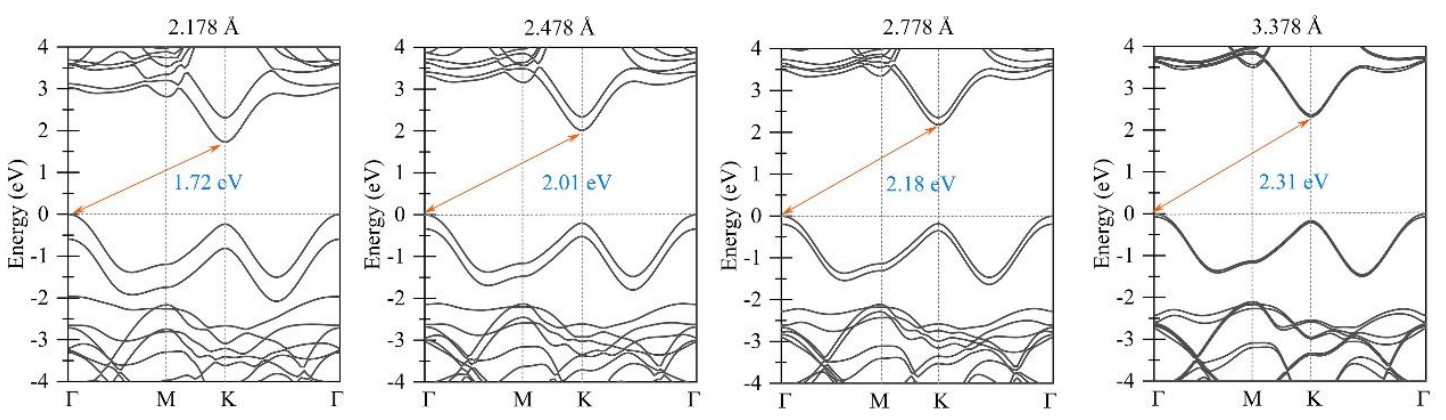

Figure S6. Band structure of bilayer $\mathrm{MoSi}_{2} \mathrm{~N}_{4}$ at different interlayer distances using HSE06 functional.

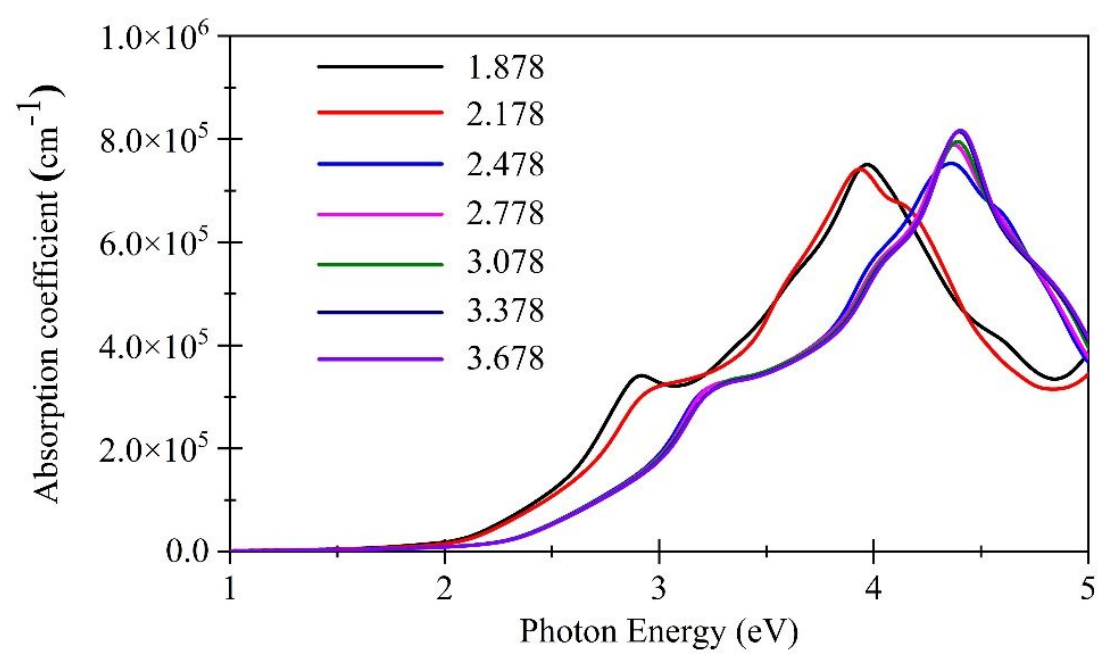

Figure S7. Optical absorption coefficients of $\mathrm{AB}-\mathrm{MoSi}_{2} \mathrm{~N}_{4}$ under different interlayer distance $d(\AA)$. 


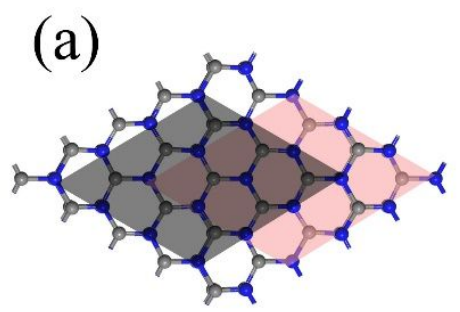

(b)
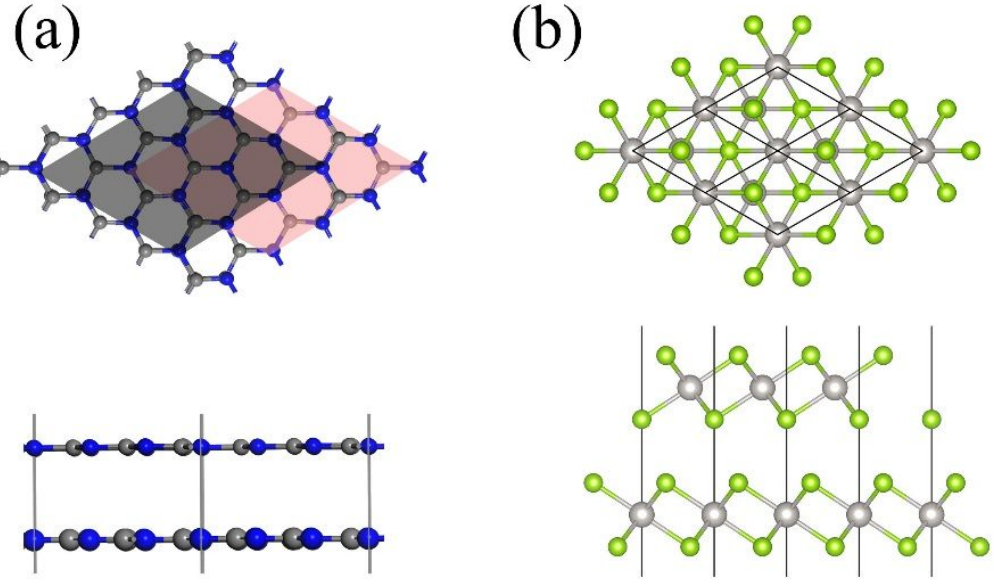

Figure S8. (a) Crystal structures of $2 \mathrm{D}$ stacking engineered (a) $\mathrm{g}-\mathrm{C}_{3} \mathrm{~N}_{4}$ and (b) $\mathrm{PtS}_{2}$ photocatalysts with built-in electric field. 